\title{
A perverse sheaf approach toward a cohomology theory for string
}

\section{theory}

\author{
Abdul Rạ̣mān \\ Department of Physics and Astronomy, Howard University, Washington, \\ DC 20059, USA \\ arahman@howard.edu
}

\begin{abstract}
We present the construction and properties of a self-dual perverse sheaf $\underline{\underline{\mathcal{S}_{0}}}$ whose cohomology fulfills some of the requirements of String theory as $\overline{\overline{\text { out }}}$ lined in [1]. The construction of this $\underline{\underline{\mathcal{S}_{0}}}$ utilizes techniques that follow from MacPherson-Vilonen [2]. Finally, we will discuss its properties as they relate to String theory.
\end{abstract}

\section{Introduction}

In analyzing ways to move between Calabi and Yau manifolds, Green and Hubsch in $[3,4]$ showed there are cases within String theory that admit mildly singular target spaces. These mildly singular spaces, termed conifolds $[3,5,6]$ consist of the usual smooth target spaces with the addition of zero-dimensional singularities that stratify these spaces. Since these spaces

e-print archive: http://lanl.arXiv.org/abs/0704.3298 
are singular, the usual techniques for calculating cohomology do not apply. Ideally, a cohomology theory for String theory would apply to both smooth and not "too" singular target spaces ${ }^{1}$ so that determination of stringy vacua could be possible in all cases. In an effort to qualify the mathematical requirements for this (co)homology theory, Hubsch proposed in [7] a working definition for a homology theory for String theory in the case of a mildly degenerate target space. This is recalled in the following definition.

Definition 1.1. Let $Y$ be a $2 n$-dimensional stratified space with a single isolated singularity, $y$. Then

$$
S H_{k}(Y)= \begin{cases}H_{k}(Y), & k>n \\ H_{n}(Y-y) \cup H_{n}(Y), & k ; \\ H_{k}(Y-y), & k<n .\end{cases}
$$

The middle dimension case, $k, H_{n}(Y-y) \cup H_{n}(Y)$ is a qualitative way of expressing the String theory requirement that the homology group contain cycles from both $H_{n}(Y-y)$ and $H_{n}(Y)$. This means that the middle dimension homology group should be larger than either $H_{n}(Y-y)$ or $H_{n}(Y)$. Many homology theories were examined to attempt to fulfill this requirement but only intersection homology ${ }^{2}$ seemed to possess most of the requirements. However, in the middle dimension, intersection homology provided fewer (co)cyclic classes than required by String theory [5]. It was concluded that $H^{k}(Y ; \underline{I C})$ seemed to be the correct choice in all degrees except $k$, where $\underline{\underline{I C}}$ is the sheaf of intersection chains defined on p.77 of ref. [9]. In this paper we will discuss the construction of a complex of sheaves $\underline{\mathcal{S}_{0}}$ ' such that $H^{k}\left(Y ; \underline{\mathcal{S}_{0}}{ }^{\circ}\right)=H^{k}(Y ; \underline{\underline{I C}})$ in all degrees $k \neq n$, but will yield more cohomology in the middle dimension as predicted in String theory [5].

\subsection{Kähler package}

As mentioned in the previous section, massless fields in superstring compactifications were identified with cohomology classes on the target space. However, a troubling consequence occurs when the target space is singular. Essentially, different cohomology theories on singular target spaces yield different results thereby making it difficult to determine which theory physics may favor. Several important characteristics of the cohomology,

\footnotetext{
${ }^{1}$ The only known criterion on the severity of singularization comes from supersymmetry, which is essential for vacuum stability [8].

${ }^{2} \mathrm{~A}$ detailed discussion can be found in [11] and [9].
} 
which correspond to the massless fields, are based on general properties of field theories, specifically, the $(2,2)$-supersymmetric two-dimensional worldsheet field theories. These properties, known as the Kähler package, should hold for singular and smooth target spaces. Let $\mathcal{Y}$ be a smooth Calabi-Yau target space. We will use Refs. [7] and [10] for the definition of the Kähler package which is stated as follows:

1. Hodge decomposition: $H^{r}(\mathcal{Y}, \mathbb{C})=\bigoplus_{p+q=r} H^{p, q}(\mathcal{Y})$.

2. Complex conjugation: $H^{(p, q)}(\mathcal{Y})=\overline{H^{(q, p)}(\mathcal{Y})}$, which follows from CPT conjugation in the world-sheet field theory.

3. Poincare duality: $\quad H^{n-p, n-q}(\mathcal{Y}) \times H^{p, q}(\mathcal{Y}) \rightarrow H^{n, n}(\mathcal{Y})$ is nondegenerate.

4. Kunneth formula: Given two topological spaces $X$ and $Y$. Then $H^{r}(X \times Y)=\bigoplus_{p+q=r} H^{p}(X) \otimes H^{q}(Y)$ which says that in a product of two spaces, harmonic forms are products of a harmonic form from one space and a harmonic form from the other space, with their degrees added.

5. Lefshetz $S L(2, \mathbb{C})$ action.

(Remark: The Lefshetz $S L(2, \mathbb{C})$ action does not have any recognizable counterpoint in the physics applications, so we ignore it for now.)

In the case that $\mathcal{Y}$ is a singular Calabi-Yau target space, String theory suggests that these properties of the Kähler package should be preserved. Hence, a cohomology theory for String theory should not only meet the cohomology requirements qualitatively outlined by Hubsch in [1], but should also meet the properties of the Kähler package whether $\mathcal{Y}$ is singular or smooth.

In this paper, we show there exists a perverse sheaf $\mathcal{S}_{0}{ }^{\cdot}$ that fulfills these cohomology requirements and satisfies one part of the Kǟhler package. Proving the remaining parts are currently open problems. In the next section, we present the mathematical tools used in this paper.

\subsection{Useful mathematical tools}

In this paper we will use the following definitions and statements.

Definition 1.2. A $2 n$-dimensional simple stratified space $Y$ is a compact topological space with one "singular" point $y \in Y$ such that

1. $Y-\{y\}$ is a smooth 2 n-dimensional manifold called the "non-singular" part of $Y$. 
2. There exists a neighborhood $U_{y}$ of the singular point $y$ whose closure is homeomorphic to the cone $c L$ over $L$, where $L$ is a compact $(n-1)$ dimensional submanifold of $Y$, by a homeomorphism $\phi: c L \rightarrow U_{y}$ such that $\phi(*)=y$ where $*$ represents the cone point. The open cone over the link will be denoted by $c^{o} L$.

Let $Y$ be a simple stratified space with $Y^{o}$ the non-singular part of $Y$. Define the inclusions $j: Y^{o} \hookrightarrow Y$, and for the singular point $y \in Y$ define the inclusion $i:\{y\} \hookrightarrow Y$. We will denote the sections of a complex of sheaves $\underline{\underline{S}}$ over an open set $U$ as as either $\Gamma(U, \underline{\underline{S}})$ or $\underline{\underline{S}}(U)$. A constructible complex of sheaves will be denoted as $\underline{\underline{K}}$. The sheaves and complexes of sheaves used in this paper will be constructible. In addition, $H_{\mathrm{c}}^{*}$ represents cohomology with compact supports.

A morphism $f: \underline{\underline{K}} \rightarrow \underline{\underline{L}}$ of complexes of sheaves is said to be a quasiisomorphism if $\underline{\underline{H}}^{n}\left(\overline{\bar{f})}: \underline{\underline{H}}^{\overline{\bar{n}}}\left(\underline{\underline{K}}^{*}\right) \rightarrow \underline{\underline{H}}^{n}(\underline{\underline{L}})\right.$ is an isomorphism for any $n$. Let $\underline{\underline{K}}$ and $\underline{\underline{L}}$ be quasi-isomorphic complexes of sheaves in an abelian category $\overline{\overline{\mathbb{A}}}$. The derived category of $\mathbb{A}, \mathcal{D}(\mathbb{A})$, is a category in which these two complexes of sheaves are isomorphic. The bounded below derived category, $\mathcal{D}_{Y}^{b}(\mathbb{A})$, of the abelian category $\mathbb{A}$ on $Y$ has objects, complexes of sheaves, that are zero below some degree. Discussions about the derived category can be found in Section 1.7 in [12], Section 1.3 in [13], or Section 4.1 in [14].

In this paper, $\mathcal{D}_{Y}^{b}$ will denote the bounded below derived category of constructible complexes sheaves of $\mathbb{Q}$-vector spaces on $Y$. It is known that $\mathcal{D}_{Y}^{b}$ has enough injective objects in the sense of Section 1.3 in Ref. [13]. Furthermore, we will restrict our treatment to the case of all local systems being the constant sheaf $\underline{\underline{\mathbb{Q}}}$ with $Y$ a simple stratified space.

A functor $\mathrm{T}$ from the category of sheaves to an abelian category $\mathbb{A}$ gives rise to a functor RT from the derived category of sheaves to the derived category of $\mathbb{A}$. We will be using the definitions of the pullback, pushforward, extension by zero, and Verdier duality functors from [14]. Let $\underline{\underline{A}} \in \mathcal{D}_{Y}^{b}$ be a complex of sheaves on $Y^{o}$. Its pushforward by $j$ is defined to be the complex of sheaves $R j_{*} \underline{\underline{A}}$ on $Y$ whose sections over an open set $U \subset Y$ are defined as $R j_{*}(\underline{\underline{A}})(U)=\underline{\underline{A}}\left(j^{-1}(U)\right)$ and the restriction from $U$ to $V \subset U$ is induced by the restriction from $j^{-1}(U)$ to $j^{-1}(V)\left[14\right.$, p. 45]. Let $\underline{\underline{B}} \in \mathcal{D}_{Y}^{b}$ be a complex of sheaves on $Y$. Its pullback, $j^{*} \underline{\underline{B}}$, is a sheaf on $Y^{o}$ such that there is an isomorphism $\operatorname{Hom}\left(j^{*} \underline{\underline{B}}, \underline{\underline{A}}\right) \simeq \operatorname{Hom}\left(\underline{\underline{B}}, j_{*} \underline{\underline{A}}\right)$. [14, p. 46].

Let $f: U \rightarrow Y$ be an open subset of $Y$, let $\underline{\underline{\mathcal{F}}} \in \mathcal{D}_{Y}^{b}$ be a complex of sheaves on $U$. The extension by zero complex of sheaves $R j ! \underline{\underline{\mathcal{F}}}$ denotes the subsheaf of $R j_{*} \underline{\underline{\mathcal{F}}}$ given by $\Gamma(V, R j ! \underline{\underline{\mathcal{F}}})=\{s \in \Gamma(U \cap V, \underline{\underline{\mathcal{F}}}) \mid \operatorname{supp}(s)$ is closed relative 
to $V$ \} where $V$ is an open subset of $U$ and $\operatorname{supp}(s)$ denotes the support of the section $s$. More details can be found in Chaps. 2 and 5 in [14].

We will need the following facts about Verdier duality. Let $Y$ and $Z$ be topological spaces and let $f: Y \rightarrow Z$ be a map. Verdier defined a map $f^{!}: \mathcal{D}_{Z}^{b} \rightarrow \mathcal{D}_{Y}^{b}$. The Verdier duality theorem is a canonical isomorphism in $\mathcal{D}_{Z}^{b}$,

$$
R f_{*} R \underline{\underline{\operatorname{Hom}}}\left(\underline{\underline{A}}^{\cdot}, f^{!} \underline{\underline{B}}\right) \simeq R \underline{\underline{\operatorname{Hom}}}\left(R f_{!} \underline{\underline{A}}^{*}, \underline{\underline{B}}^{\cdot}\right)
$$

for any $\underline{\underline{A}} \in \mathcal{D}_{Y}^{b}$ and $\underline{\underline{B}} \in \mathcal{D}_{Z}^{b}$.

Definition 1.3 (Dualizing sheaf). Let $Y$ be a locally compact topological space and let $f: Y \rightarrow\{p t\}$ be the map to a point. Verdier defined the dualizing sheaf to be $\mathbb{D}_{Y}:=f^{!} \mathbb{Q}$.

Let $Y$ be a locally compact topological space and let $\underline{\underline{A}} \in \mathcal{D}_{Y}^{b}$ be a complex of sheaves. In ref. [15], Verdier defined the duality functor $\mathcal{D}_{V}$ by $\mathcal{D}_{V}(\underline{\underline{A}}):=$ $\underline{\underline{R \operatorname{Hom}}}\left(\underline{\underline{A}}, \mathbb{D}_{Y}\right)$.

Definition 1.4 (Verdier Duality Functor). The duality functor $\mathcal{D}_{V}$ has the following properties (p. 92, [9]). Let $Y$ and $Z$ be topological spaces and let $f: Y \rightarrow Z$ be a map. There exist isomorphisms in $\mathcal{D}_{Y}^{b}$ as follows:

1. $\mathbb{D}_{Y} \cong \mathcal{D}_{V}(\mathbb{Q}) \cong f^{!}\left(\mathbb{D}_{Z}\right)$.

2. $\underline{\underline{A}} \cong \mathcal{D}_{V}\left(\mathcal{D}_{V}(\underline{\underline{A}})\right)$.

Definition 1.5 (Verdier dual pairing [15]). Let $\underline{\underline{S}}$ and $\underline{\underline{T}}$ be complexes of sheaves defined on the simple stratified space $\overline{\bar{Y}}$. A Verdier dual pairing between $\underline{\underline{S}}$ and $\underline{\underline{T}}$ is a morphism $\phi: \underline{\underline{S}} \otimes \underline{\underline{T}} \rightarrow \mathbb{D}_{Y}[-2 n]$ which induces a quasi-isomorphism $\left.\underline{\underline{S}} \rightarrow \underline{\underline{R \operatorname{Hom}}}\left(\underline{\underline{T}}, \mathbb{D}_{Y} \underline{\bar{S}}-2 n\right]\right)$ where $\mathbb{D}_{Y}$ is the dualizing sheaf on $Y$.

Proposition 1.1. Suppose $\underline{\underline{S}}$ and $\underline{\underline{T}}$ are complexes of sheaves with a Verdier dual pairing $\phi$ on the simple stratified space $Y$. Then the morphism $\phi$ induces a non-degenerate pairing on cohomology $\hat{\phi}: H^{i}(Y, \underline{\underline{S}}) \otimes H^{2 n-i}(Y, \underline{\underline{T}}) \rightarrow$ $H^{0}\left(Y, \mathbb{D}_{Y}\right) \cong \underline{\underline{Q}}$.

Two particular distinguished triangles will be used in this paper. Let $U$ be an open set in a topological space $Y$ and let $Z$ be its closed complement. Let $\underline{\underline{S}}$ be a complex of sheaves on $Y$. Then there are distinguished triangles 
defined as follows:
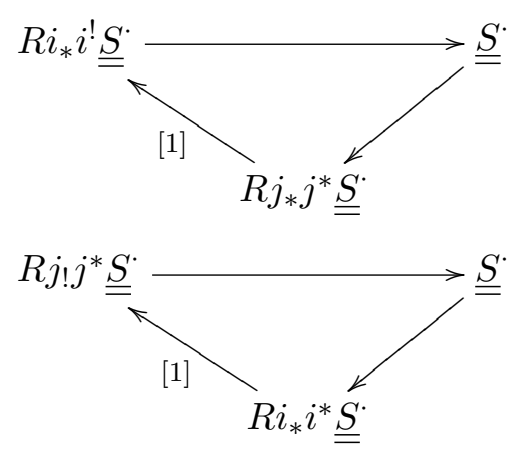

These distinguished triangles (1.3) and (1.4) induce long exact sequences on cohomology:

$$
\begin{aligned}
\cdots \rightarrow H^{n}\left(Y ; R i_{*} i^{!} \underline{\underline{S}}^{\cdot}\right) \rightarrow H^{n}(Y ; \underline{\underline{S}}) \rightarrow & H^{n}\left(Y ; R j_{*} j^{*} \underline{\underline{S}}\right) \\
& \rightarrow H^{n+1}\left(Y ; R i_{*} i^{!} \underline{\underline{S}}\right) \rightarrow \cdots
\end{aligned}
$$

and

$$
\begin{aligned}
\cdots \rightarrow H^{n}\left(Y ; R j_{!} j^{*} \underline{\underline{S}}^{\cdot}\right) \rightarrow H^{n}(Y ; \underline{\underline{S}}) \rightarrow & H^{n}\left(Y ; R i_{*} i^{*} \underline{\underline{S}}\right) \\
& \rightarrow H^{n+1}\left(Y ; R j_{!} j^{*} \underline{\underline{S}}\right) \rightarrow \cdots
\end{aligned}
$$

respectively. We will require another relation that arises through manipulation of distinguished triangles.

Lemma 1.1. For any complex of sheaves $\underline{\underline{\mathcal{P}}}$ on the simple stratified space $Y$, there is a natural isomorphism $H^{k}\left(i^{*} R \underline{\overline{j_{*}}} \underline{\underline{\mathcal{P}}}\right) \cong H^{k+1}\left(i^{!} R j ! \underline{\underline{\mathcal{P}}}\right)$.

Proof. Recall that for any complex of sheaves $\underline{\underline{\mathcal{S}}}$ we have the distinguished triangle (1.3)

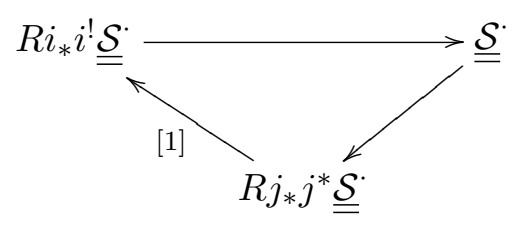


Let $\underline{\underline{\mathcal{S}}}=R j ! \underline{\underline{\mathcal{P}}}$ where $\underline{\underline{\mathcal{P}}}$ is a complex of sheaves. Then we have

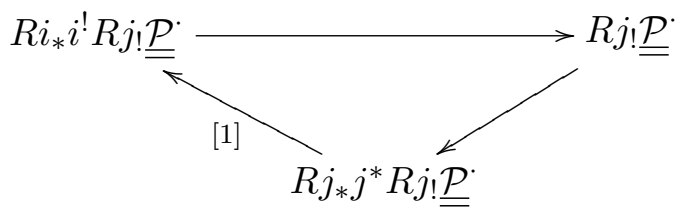

We know that $R j_{*} j^{*} R j ! \underline{\underline{\underline{P}}}=R j_{*} \underline{\underline{\mathcal{P}}}$. Substituting into equation (1.8) above we have

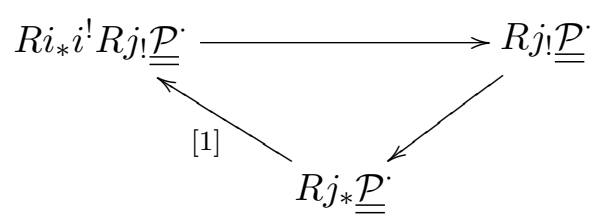

Applying $i^{*}$ to equation (1.9) we obtain

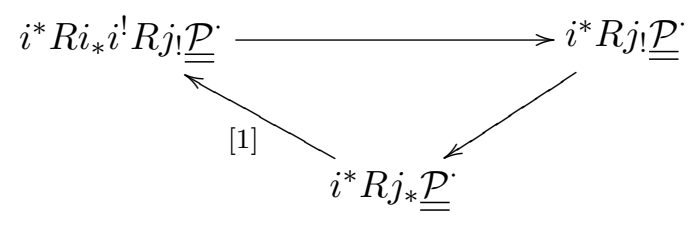

This simplifies to

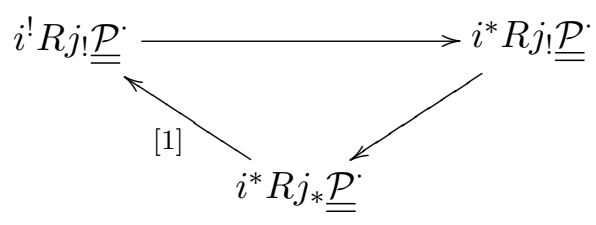

Since $R j$ ! is the extension by zero functor, it follows that $i^{*} R j ! \underline{\underline{\mathcal{P}}}=0$. Hence,

$$
i^{*} R j_{*} \underline{\underline{\mathcal{P}}} \stackrel{[1]}{\longrightarrow} i^{!} R j ! \underline{\underline{\mathcal{P}}}
$$

is an isomorphism in all degrees $k>0$. Applying $H^{*}$ to equation (1.12) we get

$$
H^{k}\left(i^{*} R j_{*} \underline{\underline{\mathcal{P}}}^{*}\right) \cong H^{k+1}\left(i^{!} R j ! \underline{\underline{\mathcal{P}^{*}}}\right)
$$

for all $k>0$. 
Lemma 1.2. Let $Y$ be a simple stratified space and let $\underline{\underline{\mathcal{P}}}$ be a complex of sheaves on $Y$. Let $y \in Y$ be the singular point and let $U_{y}$ be a distinguished neighborhood of $y$ so that $\bar{U}_{y} \simeq c L_{y}$ and $U_{y} \simeq c^{o} L_{y}$. Then there are natural isomorphisms

1. $H^{m}\left(Y ; i_{*} i^{*} \underline{\mathcal{P}}\right) \cong H^{m}\left(Y^{o} ; \underline{\mathcal{P}}\right), \forall m>0$,

2. $H^{m}\left(Y ; i_{i} i^{*} \overline{\overline{\mathcal{P}}}\right) \cong H_{\mathrm{c}}^{m}\left(Y^{o} ; \underline{\overline{\mathcal{P}}}\right), \forall m>0$,

3. $H^{m}\left(Y ; j_{*} j^{* \underline{\mathcal{P}}}\right) \cong H^{m}\left(U_{y} ; \underline{\mathcal{P}}\right), \forall m>0$,

4. $H^{m}\left(Y ; j_{*} j ! \overline{\overline{\mathcal{P}}}\right) \cong H_{\mathrm{c}}^{m}\left(U_{y} ; \underline{\underline{\underline{\mathcal{P}}}}\right), \forall m>0$,

5. $H^{m}\left(Y ; j^{*} i_{*} \underline{\overline{\mathcal{P}}}\right) \cong H^{m}(L \times(0,1) ; \underline{\underline{\mathcal{P}}}), \forall m>0$ where $L$ is the link of the singular point $y$.

Remark: The proof of the results in Lemma 1.2 follow in a straightforward manner from sheaf theory. For the remainder of this paper, $L$ and $L_{y}$ will be used interchangeably to denote the link of the point $y \in Y$. The derived category, quasi-isomorphisms, right derived functors and related topics are taken from the Refs. $[13,14,16]$. In the next section we present the motivation for constructing the perverse sheaf $\underline{\underline{\mathcal{S}_{0}}}$.

\subsection{Mathematical approach}

The objective of this paper is to construct the minimal object $\underline{\underline{\mathcal{S}_{0}}}$ using the technique of MacPherson and Vilonen [2, Thm. 2.1], show it provides the necessary cohomology in all degrees, discuss its properties, and then provide some qualitative insight to applications to String theory. The cohomology requirement for the middle dimension as stated in the Introduction can be expressed mathematically as follows. Let $Y$ be a simple stratified space. We seek a minimal object $\underline{\underline{\mathcal{S}_{0}}}$ such that $H^{n}\left(Y ; \underline{\underline{\mathcal{S}_{0}}}{ }^{\circ}\right)$ fits into the following diagram:

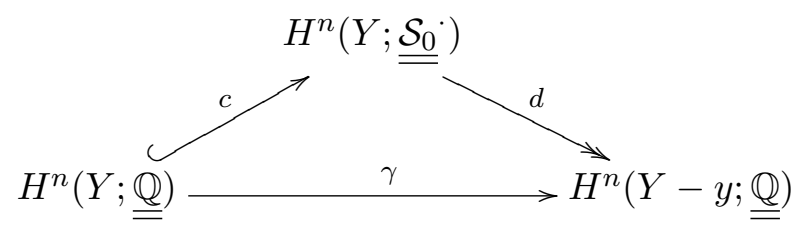

where $c$ is an injection, $d$ is a surjection, and $\gamma$ is the restriction map on cohomology. This minimal object $\underline{\mathcal{S}_{0}}$ would have cohomology greater than either $H_{n}(Y-y)$ or $H_{n}(Y)$ for $k$, but $H^{k}\left(Y ; \underline{\underline{\mathcal{S}_{0}}}\right) \cong H^{k}(Y ; \underline{\underline{I C}})$ in all other degrees. The existence and construction of such an ${\underline{\underline{\mathcal{S}_{0}}}}_{\text {that yields the }}$ desired cohomology in the case of a singular isolated point is the subject 
of this paper. In order to construct $\underline{\mathcal{S}_{0}}$ on $Y$, we will use the method developed by MacPherson and Vilonen presented in [2]. In this technique, a perverse sheaf will be constructed from datum on the non-singular part, $Y^{o}$ of $Y$. Although [2] describes how to construct perverse sheaves on stratified spaces with higher dimensional strata and non-trivial local systems defined on $Y^{o}$, we have adapted this technique for the case of a space $Y$ that has a "simple stratification" made up of two parts: the singular point $y \in Y$ and the smooth $Y^{o}$. In addition, the constant local system defined on $Y^{o}$ will be used in all cases. In the next section, the category of perverse sheaves and the zig-zag category will be presented.

\section{Perverse sheaves and the zig-zag category}

In this section we will present a condensed discussion of the category of perverse sheaves and the zig-zag category. Throughout this paper we restrict ourselves to conventions as presented in Section 1.2.

\subsection{The category of perverse sheaves}

Definition 2.1. The category of perverse sheaves $\mathbb{P}(Y)$ is the full subcategory of $\mathcal{D}^{b}(Y)$ whose objects are complexes of sheaves $\underline{\underline{\mathcal{S}}}$, which satisfy the following properties:

1. There exists $M>0 \in \mathbb{Z}$ such that $\underline{\mathcal{S}}^{i}=0 \forall i<M$ (bounded below)

2. The complex of sheaves $j^{*} \underline{\underline{\mathcal{S}}}$ is quasi-isomorphic to a local system on $Y^{o}$ (in degree 0 ). In other words,

(a) $\underline{H}^{k}\left(j^{*} \underline{\mathcal{S}}\right)=0$ if $k \neq 0$

(b) ${\underline{\underline{H^{0}}}}^{0}\left(j^{*} \underline{\underline{\mathcal{S}}}\right)$ is a local system

3. $H^{k}\left(i^{*} \underline{\underline{\mathcal{S}}}\right)=0$ for $k>n$ (support)

4. $H^{k}(i ! \underline{\underline{\mathcal{S}}})=0$ for $k<n$ (cosupport)

Remark: We will be primarily interested in the sub-category $\mathbb{P}_{\mathbb{Q}}(Y)$ of $\mathbb{P}(Y)$ that consists of perverse sheaves $\underline{\underline{\mathcal{S}}}$ where the local system $\underline{\underline{H}}^{0}\left(j^{*} \underline{\underline{\mathcal{S}}}\right)$ is the constant sheaf $\underline{\underline{Q}}$. Let $\mathbb{P}\left(Y^{o}\right)$ represent the category of perverse sheaves on $Y^{o}$. This is equivalent to the category of local systems defined on $Y^{o}$.

\section{$2.2 \quad$ Zig-zag Category}

\subsubsection{Definitions and properties}

We have modified the definition of the Zig-zag category $Z(Y, y)$ as taken from p. 409 in Ref. [2]. 
Definition 2.2 $(Z(Y, y))$. An object in $Z(Y, y)$ is a sextuple $\Theta=(\mathcal{L}, K, C$, $\alpha, \beta, \gamma)$ where $\mathcal{L} \in \mathbb{P}\left(Y^{o}\right)$, and $K$ and $C$ are vector spaces together with an exact sequence:

$$
H^{n-1}\left(i^{*} j_{*} \mathcal{L}\right) \stackrel{\alpha}{\longrightarrow} K \stackrel{\beta}{\longrightarrow} C \stackrel{\gamma}{\longrightarrow} H^{n}\left(i^{*} j_{*} \mathcal{L}\right)
$$

Let $\Theta^{\prime}=\left(\mathcal{L}^{\prime}, K^{\prime}, C^{\prime} \alpha^{\prime}, \beta^{\prime}, \gamma^{\prime}\right) \in \operatorname{Obj}(Z(Y, y))$. A morphism $\theta: \Theta \rightarrow \Theta^{\prime}$ in $Z(Y, y)$ consists of maps $\mathcal{L} \mapsto \mathcal{L}^{\prime}, K \mapsto K^{\prime}$ and $C \mapsto C^{\prime}$ such that the following diagram commutes:

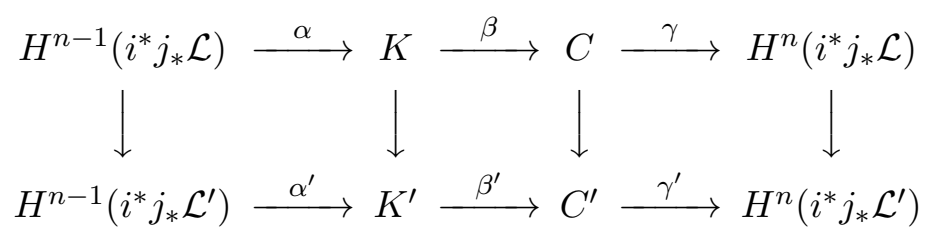

Remark: We will be primarily interested in the full sub-category $Z_{\mathbb{Q}}(Y, y)$ of $Z(Y, y)$ that consists of zig-zag objects $\Theta$ where the local system $\mathcal{L}$ is the constant sheaf $\underline{\underline{Q}}$.

Definition 2.3. The zig-zag functor $\mu: \mathbb{P}(Y) \rightarrow Z(Y, y)$ is defined by sending an object $\underline{\underline{Q}} \in \mathbb{P}(Y)$ to the triple $\left(j^{*} \underline{\underline{Q}}, H^{n}\left(i^{!} \underline{\underline{Q}}\right), H^{n}\left(i^{*} \underline{\underline{Q}}\right)\right)$ together with the exact sequence

$$
H^{n-1}\left(i^{*} j_{*} j^{*} \underline{\underline{Q^{*}}}\right) \longrightarrow H^{n}\left(i^{!} \underline{\underline{Q^{*}}}\right) \longrightarrow H^{n}\left(i^{*} \underline{\underline{Q^{*}}}\right) \longrightarrow H^{n}\left(i^{*} j_{*} j^{*} \underline{\underline{\overline{Q^{*}}}}\right)
$$

\subsubsection{Relationship to $\mathbb{P}(Y)$}

We will use Theorem 2.1 from MacPherson and Vilonen [2] in the proof of the main result. It is stated below in a modified form.

Theorem 2.1. (MacPherson-Vilonen [2])

1. The zig-zag functor $\mu: \mathbb{P}(Y) \rightarrow Z(Y, y)$ gives rise to a bijection from isomorphism classes of objects of $\mathbb{P}(Y)$ to isomorphism classes of objects of $Z(Y, y)$,

2. Given $\underline{\underline{\mathcal{S}}}, \underline{\underline{\mathcal{S}^{\prime}}} \in \mathbb{P}(Y)$. Then $\mu: \operatorname{Hom}_{\mathbb{P}}\left(\underline{\underline{\mathcal{S}}}, \underline{\underline{\mathcal{S}^{\prime}}}\right) \rightarrow \operatorname{Hom}_{Z}\left(\mu(\underline{\underline{\mathcal{S}}}), \mu\left(\underline{\underline{\mathcal{S}^{\prime}}}\right)\right)$ is a surjection.

Hence an isomorphism class of objects in $\mathbb{P}(Y)$ is determined by a local system (in this case the constant local system) on $Y^{o}$ and a fixed $K$ and $C$. 
Different choices of $K$ and $C$ lead to different perverse sheaves on $Y$. The main result of this work involves constructing a certain perverse sheaf on $Y$ from a certain choice of $K$ and $C$, as described in the following proposition.

Proposition 2.1. Let $\Theta_{0}=\left(\underline{\mathbb{Q}}, K_{0}, C_{0}, \alpha_{0}, \beta_{0}, \gamma_{0}\right)$ where $K_{0}=\operatorname{Im}\left(H_{\mathrm{c}}^{n}\left(c^{o} L\right)\right.$ $\left.\rightarrow H_{\mathrm{c}}^{n}\left(Y^{o}\right)\right), C_{0}=\operatorname{Im}\left(H^{n}\left(Y^{0}\right) \rightarrow H_{\mathrm{c}}^{n+1}\left(c^{o} L\right)\right)$. Let $\alpha_{0}: H_{\mathrm{c}}^{n}\left(c^{o} L\right) \rightarrow \operatorname{Im}\left(H_{\mathrm{c}}^{n}\right.$ $\left.\left(c^{o} L\right) \rightarrow H_{\mathrm{c}}^{n}\left(Y^{o}\right)\right)$. Let $\beta_{0}$ be the 0-map. Let $\gamma_{0}: \operatorname{Im}\left(H^{n}\left(Y^{0}\right) \rightarrow H_{\mathrm{c}}^{n+1}\left(c^{o} L\right)\right)$ $\rightarrow H_{\mathrm{c}}^{n+1}\left(c^{o} L\right)$. Then $\Theta_{0} \in \operatorname{Obj}\left(Z_{\mathbb{Q}}(Y, y)\right)$. Therefore there exists $\underline{\underline{\mathcal{S}_{0}}} \in \mathbb{P}_{\mathbb{Q}}$ (Y) such that $\mu\left(\underline{\underline{\mathcal{S}_{0}}}\right)=\Theta_{0}$.

Proof. In order to have an object of $Z_{\mathbb{Q}}(Y, y)$, we must specify $K_{0}, C_{0}$, the maps between them and then show equation (2.1) is exact. Rewriting equation (2.1) yields

$$
H_{\mathrm{c}}^{n}\left(c^{o} L\right) \stackrel{\alpha_{0}}{\longrightarrow} K_{0} \stackrel{\beta_{0}}{\longrightarrow} C_{0} \stackrel{\gamma_{0}}{\longrightarrow} H_{\mathrm{c}}^{n+1}\left(c^{o} L\right)
$$

Recall the distinguished triangle in equation (1.3) applied to $Y$ with coefficients in $\underline{\underline{\mathbb{Q}}}$. Simplifying in degree $n$ we have

$$
\begin{aligned}
& \longrightarrow H_{\mathrm{c}}^{n}\left(c^{o} L\right) \longrightarrow H_{\mathrm{c}}^{n}\left(Y^{o}\right) \longrightarrow H^{n}\left(Y^{o}\right) \\
& \longrightarrow H_{\mathrm{c}}^{n+1}\left(c^{o} L\right) \longrightarrow
\end{aligned}
$$

Comparing eqns. (2.4) and (2.5), it follows by definition that we can identify the canonical maps $\alpha_{0}: H_{c}^{n}\left(c^{o} L ; \underline{\underline{\mathbb{Q}}}\right) \rightarrow K_{0}$ as a surjection and $\gamma_{0}: C_{0} \rightarrow$ $H_{c}^{n+1}\left(c^{o} L ; \underline{\underline{Q}}\right)$ as an injection. Since equation $(2.5)$ is exact with $K_{0} \subset$ $H_{\mathrm{c}}^{n}(Y ; \underline{\underline{Q}})$ and $C_{0} \subset H_{\mathrm{c}}^{n+1}\left(c^{o} L ; \underline{\underline{Q}}\right), \beta_{0}: K_{0} \rightarrow C_{0}$ is the zero map since moving across two elements of an exact sequence is equivalent to applying $d \circ d=0$. All that remains is to show that equation (2.4) is exact. Now, since $\beta_{0}$ is the zero map it follows that $\operatorname{Ker}\left(\beta_{0}\right)=\operatorname{Im}\left(\beta_{0}\right)=0$. Since $\alpha_{0}$ is a surjection, $\operatorname{Ker}\left(\beta_{0}\right)=\operatorname{Im}\left(\alpha_{0}\right)=0$. Similarly since $\gamma_{0}$ is an injection, $\operatorname{Ker}\left(\gamma_{0}\right)=\operatorname{Im}\left(\beta_{0}\right)=0$, hence (2.4) is exact and $\Theta_{0} \in \operatorname{Obj}\left(Z_{\mathbb{Q}}(Y, y)\right)$. Since, $\Theta_{0} \in \operatorname{Obj}\left(Z_{\mathbb{Q}}(Y, y)\right)$, it follows by Theorem 2.1 there exists $\underline{\underline{\mathcal{S}_{0}}} \in \mathbb{P}_{\mathbb{Q}}(Y)$ such that $\mu\left(\underline{\underline{\mathcal{S}_{0}}}\right)=\Theta_{0}$.

\section{Main result}

In this section we state the main result and discuss its proof. 
Theorem 3.1. The perverse sheaf $\underline{\underline{\mathcal{S}_{0}}}$ has the following properties:

1. $H^{i}\left(Y ; \underline{\underline{\mathcal{S}_{0}}}\right)= \begin{cases}H^{i}(Y), & i>n, \\ H^{i}\left(Y^{o}\right), & i<n\end{cases}$

2. $H^{n}\left(Y ; \underline{\underline{\mathcal{S}_{0}}}\right)$ is specified by the following two canonical short exact sequences:

(a) $0 \rightarrow K_{0} \rightarrow H^{n}\left(Y ; \mathcal{S}_{0}\right) \rightarrow H^{n}\left(Y^{o}\right) \rightarrow 0$

(b) $0 \rightarrow H_{\mathrm{c}}^{n}\left(Y^{o}\right) \rightarrow H^{\overline{\bar{n}}} Y$; $\left.\underline{\underline{\mathcal{S}_{0}}}\right) \rightarrow C_{0} \rightarrow 0$

3. $\mathcal{S}_{0}$ is self-dual.

Remark: The two short exact sequences in (2) are equivalent to the diagram

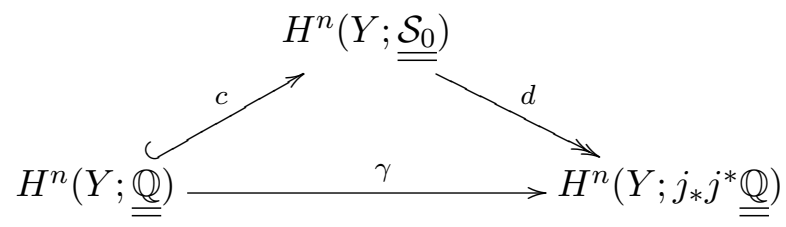

where $c$ is an injection, $d$ is a surjection, and $\gamma$ is the map that comes from the distinguished triangle between these elements.

Proof (Parts 1 and 2).

Recall that $H^{n}\left(Y ; \underline{\underline{\mathcal{S}_{0}}}\right)$ fits into the long exact sequence generated by applying the distinguished triangle (1.4) to $Y$ on $\underline{\mathbb{Q}}$. We will prove there is a diagram

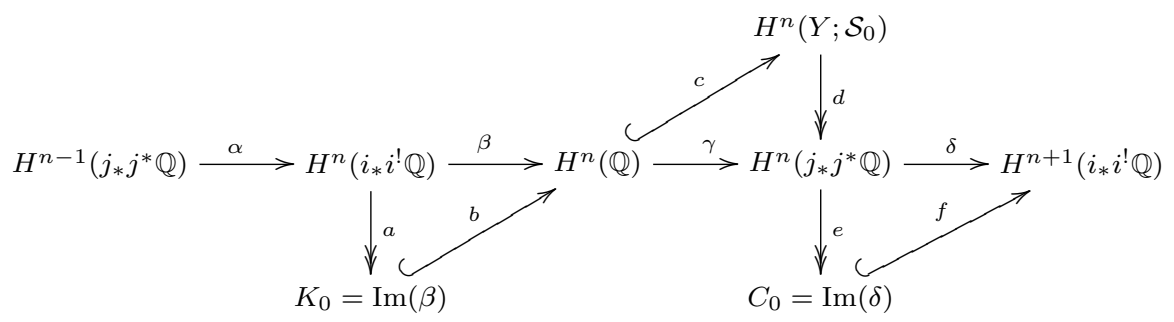

We will construct the maps for this triangle and show they exist. Recall the support and cosupport conditions for a perverse sheaf described in Definition 2.1. Consider the distinguished triangle (1.3) applied to $Y$ with values in the perverse sheaf $\underline{\underline{\mathcal{S}_{0}}}$. Explicitly, this is $H^{k}\left(Y ; i_{*} i^{!} \underline{\underline{\mathcal{S}_{0}}}\right) \rightarrow H^{k}\left(Y ; \underline{\underline{\mathcal{S}_{0}}}\right) \rightarrow$ $H^{k}\left(Y ; j_{*} j^{*} \underline{\underline{\mathcal{S}_{0}}}\right)$ (see equation $\left.(1.5)\right)$. Recall that, $H^{k}\left(Y ; \overline{\overline{j_{*} j^{*}}} \underline{\underline{\mathcal{S}_{0}}}\right) \cong H^{k}\left(\overline{\overline{Y^{o}}} ; \mathbb{Q}\right)$ 
$\simeq H^{k}\left(Y^{o}\right)$. Then applying the cosupport condition in Definition 2.1, $H^{k}$ $\left(Y ; i_{*} i \underline{\underline{\mathcal{S}_{0}}}\right)=0$ which implies that

$$
H^{i}\left(Y ; \underline{\underline{\mathcal{S}_{0}}}\right) \simeq H^{i}\left(Y^{o}\right)(0<i<n-1) .
$$

Consider once again the distinguished triangle (1.3) applied to $Y$ with values in the perverse sheaf $\underline{\underline{\mathcal{S}_{0}}}$ in the following degrees:

$$
\begin{aligned}
& H^{n-1}\left(Y ; i_{*} i^{\prime} \underline{\underline{\mathcal{S}_{0}}}\right) \longrightarrow H^{n-1}\left(Y ; \underline{\underline{\mathcal{S}_{0}}}\right) \longrightarrow H^{n-1}\left(Y ; j_{*} j^{*} \underline{\underline{\mathcal{S}_{0}}}\right) \\
& \longrightarrow H^{n}\left(Y ; i_{*} i^{\underline{\underline{\mathcal{S}_{0}}}}\right)
\end{aligned}
$$

By the cosupport condition in Definition $2.1, H^{n-1}\left(Y ; i_{*} i \underline{\underline{\mathcal{S}_{0}}}\right)=0$. So (3.4) becomes

$$
0 \longrightarrow H^{n-1}\left(Y ; \underline{\underline{\mathcal{S}_{0}}}\right) \longrightarrow H^{n-1}\left(Y ; j_{*} j^{*} \underline{\underline{\mathcal{S}_{0}}}\right) \longrightarrow H^{n}\left(Y ; i_{*} i^{!} \underline{\underline{\mathcal{S}_{0}}}\right)
$$

Identifying the maps in (3.5) we have

$$
0 \longrightarrow H^{n-1}\left(Y ; \underline{\underline{\mathcal{S}_{0}}}\right) \stackrel{\theta}{\longrightarrow} H^{n-1}\left(Y ; j_{*} j^{*} \underline{\underline{\mathcal{S}_{0}}}\right) \stackrel{\phi}{\longrightarrow} H^{n}\left(Y ; i_{*} i^{!} \underline{\underline{\mathcal{S}_{0}}}\right)
$$

By inspection of (3.6), $\theta$ is an injection. Recall that $H^{n}\left(Y ; i_{*} i^{!} \underline{\mathcal{S}_{0}}\right)=H_{\mathrm{c}}^{n}$ $\left(c^{o} L ; \underline{\underline{\mathcal{S}_{0}}}\right)=K_{0}$. So $(3.6)$ can be rewritten as

$$
0 \longrightarrow H^{n-1}\left(Y ; \underline{\underline{\mathcal{S}_{0}}}\right) \stackrel{\theta}{\longrightarrow} H^{n-1}\left(Y ; j_{*} j^{*} \underline{\underline{\mathcal{S}_{0}}}\right) \stackrel{\phi}{\longrightarrow} K_{0}
$$

Recall Proposition 2.1, where we defined $K_{0}=\operatorname{Im}(\beta) \subset H^{n}(\mathbb{Q})$. This implies that $\phi=\alpha \circ \beta=0$ since the horizontal part of (3.2) is part of a long exact sequence. Hence $\phi$ is the zero map which means that $\theta$ is an isomorphism. The result is that $H^{n-1}\left(Y ; \underline{\underline{\mathcal{S}_{0}}}\right) \simeq H^{n-1}\left(Y ; j_{*} j^{*} \underline{\underline{\mathcal{S}_{0}}}\right)$. This means that (3.3) can be rewritten to include $\overline{\overline{\text { degree }}} n-1$ as

$$
H^{i}\left(Y ; \mathcal{S}_{0}\right) \simeq H^{i}\left(Y^{o}\right)(0<i \leq n-1) .
$$

Consider the distinguished triangle (1.4) applied to $Y$ with values in the perverse sheave $\underline{\underline{\mathcal{S}_{0}}}$. Then for $i>n+1$, by the support condition in Definition $2.1, H^{k}\left(Y ; \overline{i *}_{*}^{*} \underline{\underline{\mathcal{S}_{0}}}\right)=0$, which implies that $H^{k}\left(Y ; \underline{\underline{\mathcal{S}_{0}}}\right) \simeq H^{k}\left(Y ; j ! j^{*} \underline{\underline{\mathcal{S}_{0}}}\right) \simeq$ $H_{\mathrm{c}}^{k}\left(Y^{o}\right) \simeq H^{k}\left(Y, c^{o} \overline{\bar{L}} \underline{\underline{\mathcal{S}_{0}}}\right) \simeq H^{k}(Y)$. It follows that

$$
H^{i}\left(Y ; \underline{\underline{\mathcal{S}_{0}}}\right) \simeq H^{i}(Y)(i>n+1)
$$


Consider once again the distinguished triangle (1.4) applied to $Y$ with values in the perverse sheaf $\underline{\underline{\mathcal{S}_{0}}}$ in the following degrees:

$$
\begin{aligned}
& H^{n}\left(Y ; i_{*} i^{*} \underline{\underline{\mathcal{S}_{0}}}\right) \longrightarrow H^{n+1}\left(Y ; j ! j^{*} \underline{\underline{\mathcal{S}_{0}}}\right) \longrightarrow H^{n+1}\left(Y ; \underline{\underline{\mathcal{S}_{0}}}\right) \\
& \longrightarrow H^{n+1}\left(Y ; i_{*} i^{*} \underline{\underline{\mathcal{S}_{0}}}\right)
\end{aligned}
$$

By the support condition in Definition 2.1, $H^{n+1}\left(Y ; i_{*} i^{*} \underline{\underline{\mathcal{S}_{0}}}\right)=0$. So (3.10) becomes

$$
H^{n}\left(Y ; i_{*} i^{*} \underline{\underline{\mathcal{S}_{0}}}\right) \longrightarrow H^{n+1}\left(Y ; j j^{*} \underline{\underline{\mathcal{S}_{0}}}\right) \longrightarrow H^{n+1}\left(Y ; \underline{\underline{\mathcal{S}_{0}}}\right) \longrightarrow 0
$$

Identifying the maps in (3.11) we have

$$
H^{n}\left(Y ; i_{*} i^{*} \underline{\underline{\mathcal{S}_{0}}}\right) \stackrel{\omega}{\longrightarrow} H^{n+1}\left(Y ; j_{!} j^{*} \underline{\underline{\mathcal{S}_{0}}}\right) \stackrel{\tau}{\longrightarrow} H^{n+1}\left(Y ; \underline{\underline{\mathcal{S}_{0}}}\right) \longrightarrow 0
$$

By inspection of $(3.12) \tau$ is a surjection. Recall that $H^{n}\left(Y ; i_{*} i^{*} \underline{\underline{\mathcal{S}_{0}}}\right)=H^{n}$ $\left(c^{o} L ; \underline{\underline{\mathcal{S}_{0}}}\right)=C_{0}$.

$$
C_{0} \stackrel{\omega}{\longrightarrow} H^{n+1}\left(Y ; j ! j^{*} \underline{\underline{\mathcal{S}_{0}}}\right) \stackrel{\tau}{\longrightarrow} H^{n+1}\left(Y ; \underline{\underline{\mathcal{S}_{0}}}\right) \longrightarrow 0
$$

Recall Proposition 2.1, where we defined $C_{0}=\operatorname{Im}(\delta)$. Define $\mu: C_{0} \rightarrow H^{n+1}$ $\left(Y ; \underline{\underline{\mathcal{S}_{0}}}\right)$. By exactness it follows that $C_{0}=\operatorname{Ker}(\mu)=\operatorname{Im}(\delta)$ which is a subgroup of $H^{n+1}\left(i_{*} i ! \mathbb{Q}\right)$. Since every element in $C_{0}$ maps to 0 in $H^{n+1}$ $\left(Y ; j_{!} j^{*} \underline{\underline{\mathcal{S}_{0}}}\right) \cong H_{\mathrm{c}}^{n+1}\left(Y^{o}\right) \cong H^{n+1}\left(Y ; \underline{\underline{\mathcal{S}_{0}}}\right)$ the map from $\mu$ is the 0-map and thus $\tau$ is a bijection. It follows that, we can rewrite (3.9) as

$$
H^{i}\left(Y ; \underline{\underline{\mathcal{S}_{0}}}\right) \simeq H^{i}(Y)(i \geq n+1) .
$$

Identifying the maps in the diagram (3.2), we have

$$
0 \longrightarrow K_{0} \stackrel{c \cdot b}{\longrightarrow} H^{n}\left(\underline{\underline{\mathcal{S}_{0}}}\right) \stackrel{d}{\longrightarrow} H^{n}\left(j_{*} j^{*} \mathbb{Q}\right) \longrightarrow 0
$$

We must show exactness at each term. Since $c \cdot b$ is injective we have exactness at $K_{0}$. Since $d$ is surjective we have exactness at $H^{n}\left(j_{*} j^{*} \underline{\mathbb{Q}}\right)$. All that remains is to show exactness at $H^{n}\left(\underline{\underline{\mathcal{S}_{0}}}\right)$. Recall that $K_{0}=\underline{\overline{\operatorname{Ker}}}(\gamma)$. Take $x \in K_{0}$, then $d((c \cdot b)(x))=\gamma(x)=0$. This shows that $\operatorname{Im}(c \cdot b) \subset \operatorname{Ker}(d)$. It remains to show that $\operatorname{Ker}(d) \subset \operatorname{Im}(c \cdot b)$. Take $x \in \operatorname{Ker}(d)$. We want to show $\exists y \in H^{n}(\mathbb{Q})$ such that $(c \cdot b)(y)=x$. Let $y \in H^{n}(\mathbb{Q}), x \in \operatorname{Ker}(d)$, and take $\gamma(y)=d(x)$. Recall that we required the map $d$ to be surjective, 
$(c \cdot b)(y)=x$ which means that $\operatorname{Ker}(d) \subset \operatorname{Im}(c \cdot b)$ hence, we have exactness at $H^{n}\left(\mathcal{S}_{0}\right)$.

Identifying the maps in the diagram (3.2), we have

$$
0 \longrightarrow H^{n}(\mathbb{Q}) \stackrel{c}{\longrightarrow} H^{n}\left(\mathcal{S}_{0}\right) \stackrel{e \cdot d}{\longrightarrow} C_{0} \longrightarrow 0
$$

We must show exactness at each term. Since $c$ is an injection we have exactness at $H^{n}(\mathbb{Q})$ and since $e \cdot d$ is surjective we have exactness at $C_{0}$. All that remains is to show exactness at $H^{n}\left(\underline{\underline{\mathcal{S}_{0}}}\right)$. Recall that $C_{0}=\operatorname{Im}(\delta)=\operatorname{Ker}(\mu)$ which is a subgroup of $H^{n+1}\left(i_{*} i ! \mathbb{Q}\right)$. Take $x \in H^{n}(\mathbb{Q})$ then $(e \cdot d)(c(x))=$ $\delta(\gamma(x))=0$. This shows that $\operatorname{Im}(c) \subset \operatorname{Ker}(e \cdot d)$. It remains to show that $\operatorname{Ker}(e \cdot d) \subset \operatorname{Im}(c)$. Take $y \in \operatorname{Ker}(e \cdot d)$. We want to show $\exists z \in H^{n}(\mathbb{Q})$ such that $c(z)=y$. Let $z \in H^{n}(\mathbb{Q}), y \in \operatorname{Ker}(e \cdot d)$, and take $\delta \cdot \gamma(z)=e \cdot d(y)$. The map $e \cdot d$ is surjective since both $e$ and $d$ are surjective. Then $y=c(z)$ which means that $\operatorname{Ker}(e \cdot d) \subset \operatorname{Im}(c)$ hence we have exactness at $H^{n}\left(\underline{\underline{\mathcal{S}_{0}}}\right)$. This completes the proof of parts 1 and 2 .

In the next section we will present the proof of part 3 of Theorem 3.1.

\section{Duality}

\subsection{Overview and definitions}

The goal of this section is to prove part 3 of the main result. We will use part 2 of Theorem 2.1 (MacPherson-Vilonen) to prove that $\underline{\mathcal{S}}_{0}$ is self-dual in $P_{\mathbb{Q}}(Y)$. The organization of this section will be around two main themes. The first goal will be to construct a duality functor in $Z(Y, y)$ and then show that the object defined in Definition 2.1 is self-dual. Then Theorem 2.1 will generate the corresponding self-dual object in $\mathbb{P}(Y)$. Recall that we will use definitions and statements from Section 1.2.

\subsection{Duality in $Z(Y, y)$}

We seek a duality functor $\mathcal{D}_{Z}$ in $Z(Y, y)$, that is compatible with the zigzag functor $\mu: \mathbb{P}(Y) \rightarrow Z(Y, y)$ stated in Definition 2.3. We will need the following lemma.

Lemma 4.1. Given $\Theta=(\mathcal{L}, K, C, \alpha, \beta, \gamma) \in \operatorname{Obj}(Z(Y, y))$. Then the morphism $\hat{\phi}$ from Proposition 1.1 gives rise to isomorphisms: 
1. $H^{n-1}\left(i^{*} j_{*} \mathcal{L}^{*}[-2 n]\right) \cong\left(H^{n}\left(i^{*} j_{*} \mathcal{L}\right)\right)^{*}$

2. $H^{n}\left(i^{*} j_{*} \mathcal{L}^{*}[-2 n]\right) \cong\left(H^{n-1}\left(i^{*} j_{*} \mathcal{L}\right)\right)^{*}$

where $\mathcal{D}_{V}(\mathcal{L})=\mathcal{L}^{*}$ and ()$^{*}$ is the vector space dual.

Remark: The following proof utilizes calculations from pp. 185-186, Lemma 2.20 in Section 20 from Ref. [17].

Proof. The morphism $\hat{\phi}$ of Proposition 1.1 gives rise to the following:

$$
\begin{aligned}
H^{n-1}\left(i^{*} j_{*} \mathcal{L}^{*}[-2 n]\right) & \cong H^{n-1}\left(i^{*} \mathcal{D}_{V}\left(j_{!} \mathcal{L}\right)\right)[-2 n] \\
& \cong H^{n-1}\left(\mathcal{D}_{V}\left(i^{!} j_{!} \mathcal{L}\right)\right)[-2 n] \\
& \cong H^{n+1}\left(\mathcal{D}_{V}\left(i^{!} j_{!} \mathcal{L}\right)\right) \\
& \cong\left(H^{n}\left(i^{*} j_{*} \mathcal{L}\right)\right)^{*}
\end{aligned}
$$

where $H^{n+1}\left(\mathcal{D}_{V}\left(i^{!} j ! \mathcal{L}\right)\right) \cong\left(H^{n}\left(i^{*} j_{*} \mathcal{L}\right)\right)^{*}$ using Lemma 1.1 .

$$
\begin{aligned}
H^{n}\left(i^{*} j_{*} \mathcal{L}^{*}[-2 n]\right) & \cong H^{n}\left(i^{*} \mathcal{D}_{V}\left(j_{!} \mathcal{L}\right)[-2 n]\right) \\
& \cong H^{n}\left(\mathcal{D}_{V}\left(i^{!} j_{!} \mathcal{L}\right)\right)[-2 n] \\
& \cong H^{n}\left(\mathcal{D}_{V}\left(i^{!} j_{!} \mathcal{L}\right)\right) \\
& \cong\left(H^{n-1}\left(i^{*} j_{*} \mathcal{L}\right)\right)^{*}
\end{aligned}
$$

where $H^{n}\left(\mathcal{D}_{V}\left(i^{!} j_{!} \mathcal{L}\right)\right) \cong\left(H^{n-1}\left(i^{*} j_{*} \mathcal{L}\right)\right)^{*}$ using Lemma 1.1 .

We now define the dual of an object in $Z(Y, y)$.

Definition 4.1. Given $\Theta=(\mathcal{L}, K, C, \alpha, \beta, \gamma) \in \operatorname{Obj}(Z(Y, y))$. Define $\mathcal{D}_{Z}(\Theta)$ $=\left(\mathcal{L}^{*}, C^{*}, K^{*}, \gamma^{*}, \beta^{*}, \alpha^{*}\right)$ where $\gamma^{*}: H^{n-1}\left(i^{*} j_{*} \mathcal{L}^{*}\right) \rightarrow C^{*}$ is the map dual to $\alpha, \beta^{*}: C^{*} \rightarrow K^{*}$ is the map dual to $\beta$, and $\alpha^{*}: K^{*} \rightarrow H^{n}\left(i^{*} j_{*} \mathcal{L}^{*}\right)$ is the map dual to $\gamma$.

Consider the object $\Theta=(\mathcal{L}, K, C, \alpha, \beta, \gamma)$ with its exact sequence and maps identified as follows:

$$
H^{n-1}\left(i^{*} j_{*} \mathcal{L}\right) \stackrel{\alpha}{\longrightarrow} K \stackrel{\beta}{\longrightarrow} C \stackrel{\gamma}{\longrightarrow} H^{n}\left(i^{*} j_{*} \mathcal{L}\right)
$$


Lemma 4.2. Let $\Theta=(\mathcal{L}, K, C, \alpha, \beta, \gamma) \in \operatorname{Obj}(Z(Y, y))$ and $\mathcal{D}_{Z}(\Theta)=$ $\left(\mathcal{L}^{*}, C^{*}, K^{*}, \gamma^{*}, \beta^{*}, \alpha^{*}\right)$ with the following maps:

$$
H^{n-1}\left(i^{*} j_{*} \mathcal{L}^{*}\right) \stackrel{\gamma^{*}}{\longrightarrow} C^{*} \stackrel{\beta^{*}}{\longrightarrow} K^{*} \stackrel{\alpha^{*}}{\longrightarrow} H^{n}\left(i^{*} j_{*} \mathcal{L}^{*}\right)
$$

where $\alpha^{*}, \beta^{*}$, and $\gamma^{*}$ are the dual maps defined in Definition 4.1. Then the sequence (4.4) is exact and it follows that $\mathcal{D}_{Z}(\Theta)$ is an object of $Z(Y, y)$.

Proof. The dual of the exact sequence in (4.3) is given by the exact sequence:

$$
\left(H^{n}\left(i^{*} j_{*} \mathcal{L}\right)\right)^{*} \stackrel{\gamma^{*}}{\longrightarrow} C^{*} \stackrel{\beta^{*}}{\longrightarrow} K^{*} \stackrel{\alpha^{*}}{\longrightarrow}\left(H^{n-1}\left(i^{*} j_{*} \mathcal{L}\right)\right)^{*}
$$

By Lemma 4.1 , since $\left(H^{n}\left(i^{*} j_{*} \mathcal{L}\right)\right)^{*} \cong H^{n-1}\left(i^{*} j_{*} \mathcal{L}^{*}\right)$ and $\left(H^{n-1}\left(i^{*} j_{*} \mathcal{L}\right)\right)^{*} \cong$ $H^{n}\left(i^{*} j_{*} \mathcal{L}^{*}\right)$, we can substitute these into (4.5) which becomes exactly (4.4). Since the sequence is exact it follows that $\mathcal{D}_{Z}(\Theta) \in \operatorname{Obj}(Z(Y, y))$.

The duality functor $\mathcal{D}_{Z}$ in $Z(Y, y)$ needs to be compatible the Verdier dual in $\mathbb{P}(Y)$. The following proposition demonstrates this.

Proposition 4.1. Let $\mu$ be the map as defined in Theorem 2.1. Then the following diagram commutes up to canonical isomorphism:

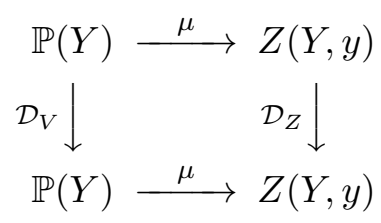

Proof. Let $\underline{\underline{\mathcal{S}}} \in \operatorname{Obj}(\mathbb{P}(Y))$. We want to show that $\mu\left(\mathcal{D}_{V}(\underline{\underline{\mathcal{S}}})\right) \cong \mathcal{D}_{Z}(\mu(\underline{\underline{\mathcal{S}}}))$. Applying $\mu$ to $\underline{\underline{\mathcal{S}}}$ gives an object in $Z(Y, y)$ which by Definition 2.3 is a triple, $\left(j^{*} \underline{\underline{\mathcal{S}}}, H^{n}\left(i^{!} \underline{\underline{\mathcal{S}}}\right), H^{n}\left(i^{*} \underline{\underline{\mathcal{S}}}\right)\right)$. This triple has an associated exact sequence obtained by applying $i^{*}$ to the distinguished triangle (1.3),

$$
H^{n-1}\left(i^{*} j_{*} j^{*} \underline{\underline{\mathcal{S}}}\right) \longrightarrow H^{n}\left(i^{!} \underline{\underline{\mathcal{S}}}\right) \longrightarrow H^{n}\left(i^{*} \underline{\underline{\mathcal{S}}}\right) \longrightarrow H^{n}\left(i^{*} j_{*} j^{*} \underline{\underline{\mathcal{S}}}\right)
$$

Applying $\mathcal{D}_{Z}$ to the zig-zag object $\mu(\underline{\underline{\mathcal{S}}})$ makes the maps in equation (4.7) reverse direction with each term dualized. The resulting zig-zag object, $\mathcal{D}_{Z}(\mu(\underline{\underline{\mathcal{S}}}))$, can be expressed as $\left(\left(j^{*} \underline{\underline{\mathcal{S}}}\right)^{*},\left(H^{n}\left(i^{*} \underline{\underline{\mathcal{S}}}\right)\right)^{*},\left(H^{n}\left(i^{!} \underline{\underline{\mathcal{S}}}\right)\right)^{*}\right)$ which 
has the following exact sequence:

$$
\begin{aligned}
\left(H^{n}\left(i^{*} j_{*} j^{*} \underline{\underline{\mathcal{S}}}\right)\right)^{*} & \longrightarrow\left(H^{n}\left(i^{*} \underline{\underline{\mathcal{S}}}\right)\right)^{*} \longrightarrow\left(H^{n}\left(i^{!} \underline{\underline{\mathcal{S}}}\right)\right)^{*} \\
& \longrightarrow\left(H^{n-1}\left(i^{*} j_{*} j^{*} \underline{\underline{\mathcal{S}}}\right)\right)^{*}
\end{aligned}
$$

Now consider the Zig-zag object $\mu\left(\mathcal{D}_{V}(\underline{\underline{\mathcal{S}}})\right)$. This is a triple $\left(j^{*} \mathcal{D}_{V}(\underline{\underline{\mathcal{S}}})\right.$, $H^{n}\left(i^{!} \mathcal{D}_{V}(\underline{\underline{\mathcal{S}}}), H^{n}\left(i^{*} \mathcal{D}_{V}(\underline{\underline{\mathcal{S}}})\right)\right.$ with exact sequence

$$
\begin{aligned}
H^{n-1}\left(i^{*} j_{*} j^{*} \mathcal{D}_{V}(\underline{\underline{\mathcal{S}}})\right) & \longrightarrow H^{n}\left(i^{\prime} \mathcal{D}_{V}(\underline{\underline{\mathcal{S}}})\right) \longrightarrow H^{n}\left(i^{*} \mathcal{D}_{V}(\underline{\underline{\mathcal{S}}})\right) \\
& \longrightarrow H^{n}\left(i^{*} j_{*} j^{*} \mathcal{D}_{V}(\underline{\underline{\mathcal{S}}})\right)
\end{aligned}
$$

Simplifying the object and the exact sequence in equation (4.9) using Lemma 4.1 we have the triple $\left(\left(j^{*} \underline{\underline{\mathcal{S}}}\right)^{*},\left(H^{n}\left(i^{*} \underline{\underline{\mathcal{S}}}\right)\right)^{*},\left(H^{n}(i \underline{\underline{\mathcal{S}}})\right)^{*}\right)$ with exact sequence

$$
\begin{aligned}
\left(H^{n}\left(i^{*} j_{*} j^{*} \underline{\underline{\mathcal{S}}}\right)\right)^{*} & \longrightarrow\left(H^{n}\left(i^{*} \underline{\underline{\mathcal{S}}}\right)\right)^{*} \longrightarrow\left(H^{n}\left(i^{!} \underline{\underline{\mathcal{S}}}\right)\right)^{*} \\
& \longrightarrow\left(H^{n-1}\left(i^{*} j_{*} j^{*} \underline{\underline{\mathcal{S}}}\right)\right)^{*}
\end{aligned}
$$

which is exactly the same as the zig-zag object $\mathcal{D}_{Z}(\mu(\underline{\mathcal{S}}))$. Showing the diagram commutes on morphisms is a similar argument and will be left to the reader.

We will need the following lemma from linear algebra.

Lemma 4.3 (Duals of Images). Given $A, B, C$, and $D$ are vector spaces with maps $f: A \rightarrow B$ and $g: C \rightarrow D$. Let $\langle,\rangle_{1}: A \times D \rightarrow \mathbb{Q}$ and $\langle,\rangle_{2}: B \times$ $C \rightarrow \mathbb{Q}$ be non-degenerate pairings such that $<a, g(c)>_{1}=<f(a), c>_{2} \forall a \in$ $A$ and $\forall c \in C$. Then $\langle,\rangle_{1}$ and $\langle,\rangle_{2}$ induce a non-degenerate pairing $\langle,\rangle_{3}$ : $\operatorname{Im}(f) \times \operatorname{Im}(g) \rightarrow \mathbb{Q}$.

Theorem 4.1. The object $\Theta_{0}$ as defined in Proposition 2.1 is self-dual in $Z_{\mathbb{Q}}(Y, y)$.

Proof. We need to construct an isomorphism between $\Theta_{0}$ and its dual $\mathcal{D}_{Z}\left(\Theta_{0}\right)$ in $Z_{\mathbb{Q}}(Y, y)$ where $\Theta_{0}=\left(\underline{\underline{Q}}, K_{0}, C_{0}, \alpha_{0}, \beta_{0}, \gamma_{0}\right)$ and $\mathcal{D}_{Z}\left(\Theta_{0}\right)=\left(\underline{\underline{Q}}^{*}\right.$, $\left.C_{0}^{*}, K_{0}^{*}, \alpha_{0}^{*}, \beta_{0}^{*}, \gamma_{0}^{*}\right)$. In order to construct an isomorphism in $Z_{\mathbb{Q}}(Y, y)$, recall the definition of a morphism in $Z_{\mathbb{Q}}(Y, y)$ (equation $(2.2)$ ) as a map from $\Theta_{0} \rightarrow \mathcal{D}_{Z}\left(\Theta_{0}\right)$ where the exact sequence for $\Theta_{0}$ maps isomorphically to the exact sequence for $\mathcal{D}_{Z}\left(\Theta_{0}\right)$ and $\underline{\mathbb{Q}}$ maps isomorphically to $\underline{\mathbb{Q}^{*}}$. These exact 
sequence maps are labelled in the following diagram:

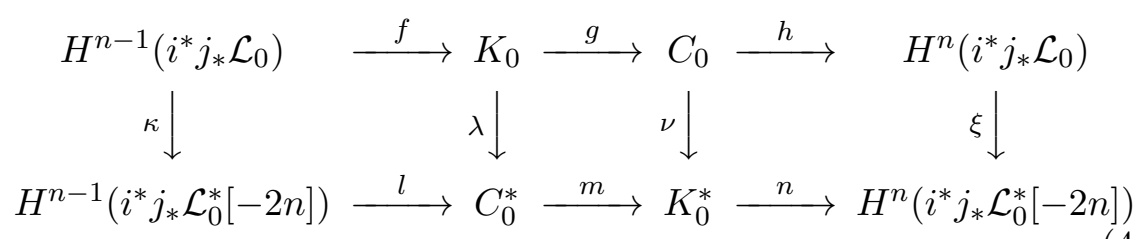

where the maps $\kappa, \lambda, \nu$, and $\xi$ are non-degenerate pairings of isomorphism classes defined as follows: $\kappa:\langle,\rangle_{\kappa}, \lambda:\langle,\rangle_{\lambda}, \nu:\langle,\rangle_{\nu}$, and $\xi:\langle,\rangle_{\xi}$. Before we begin constructing the vertical isomorphisms defined in (4.11), we must show that there is a map from $\mathbb{Q} \rightarrow \mathbb{Q}^{*}$. The map $\mathbb{Q} \otimes \mathbb{Q} \rightarrow \mathbb{Q}$ can be described by multiplication which implies that $\underline{\underline{Q}}$ is dual to itself. $\overline{\bar{T}}$ he remainder of the proof will be concerned with showing that the vertical maps $\kappa, \lambda, \nu$, and $\xi$ between the exact sequences are isomorphisms and that the diagram (4.11) commutes. By Lemma 4.1, $\kappa$ and $\xi$ are isomorphisms. Next, we must show that $\lambda$ and $\nu$ are isomorphisms. Recall that $K_{0}=\operatorname{Im}\left(H_{\mathrm{c}}^{n}\left(c^{o} L\right) \rightarrow\right.$ $\left.H_{\mathrm{c}}^{n}\left(Y^{0}\right)\right)$ and $C_{0}=\operatorname{Im}\left(H^{n}\left(Y^{0}\right) \rightarrow H_{\mathrm{c}}^{n+1}\left(c^{o} L\right)\right)$ are vector spaces. Using Proposition 1.1 we have duality isomorphisms $H_{\mathrm{c}}^{n}\left(c^{o} L\right)$ to $H_{\mathrm{c}}^{n+1}\left(c^{o} L\right)$ and $H^{n}\left(Y^{0}\right)$ to $H_{\mathrm{c}}^{n}\left(Y^{0}\right) .^{3}$ This implies there are two non-degenerate pairings $\langle,\rangle_{1}: H_{\mathrm{c}}^{n}\left(c^{o} L\right) \times H_{\mathrm{c}}^{n+1}\left(c^{o} L\right) \rightarrow \mathbb{Q}$ and $\langle,\rangle_{2}: H^{n}\left(Y^{0}\right) \times H_{\mathrm{c}}^{n}\left(Y^{0}\right) \rightarrow \mathbb{Q}$. By use of Lemma 4.3 on duals of images, $K_{0} \times C_{0} \rightarrow \mathbb{Q}$ is a non-degenerate pairing and $\lambda: K_{0} \rightarrow C_{0}^{*}$ and $\nu: C_{0} \rightarrow K_{0}^{*}$ are isomorphisms. Hence, $f$ is the dual map to $n, g$ is the dual map to $m$, and $h$ is the dual map to $l$.

It remains to show that the diagram commutes. In order to show the diagram commutes, let $x \in H^{n-1}\left(i^{*} j_{*} \mathcal{L}_{0}\right)$ and $c \in C_{0}$. We want to show that $\lambda(f(x))(c)=l(\kappa(x))(c)$. This is the same as showing $\langle f(x), c\rangle_{\lambda}=\langle x, h(c)\rangle_{\kappa}$ $\forall x \in H^{n-1}\left(i^{*} j_{*} \mathcal{L}_{0}\right)$ and $c \in C_{0}$. Since $\lambda$ is a map of isomorphism classes, $\forall$ $y \in C_{0}^{*}$ the lift of $\langle f(x), y\rangle_{\lambda}$ is $f(x)$. Recall that $h=l^{*}$. Consider $\langle x, h(c)\rangle_{\kappa} \in$ $C_{0}^{*}$. Then since $h: C_{0} \rightarrow H^{n}\left(i^{*} j_{*} \mathcal{L}_{0}\right)$, for $a \in H^{n-1}\left(i^{*} j_{*} \mathcal{L}_{0}^{*}\right), h(c)=a=$ $l^{*}(a)$. So $l\left(\langle x, a\rangle_{\kappa}\right)=\left\langle x, l^{*}(a)\right\rangle_{\kappa}=\langle x, h(c)\rangle_{\kappa}$ where $\langle x, a\rangle_{\kappa} \in H^{n-1}\left(i^{*} j_{*} \mathcal{L}_{0}^{*}\right)$. It follows that a lift of $\langle x, a\rangle_{\kappa}$ under $\kappa$ is $x$. This shows that $\lambda(f(x))(c)=$ $l(\kappa(x))(c)$.

Next we must show that for $k^{\prime}, k \in K_{0}, m(\lambda(k))\left(k^{\prime}\right)=\nu(g(k))\left(k^{\prime}\right)$. This is equivalent to showing that $\left\langle k, g\left(k^{\prime}\right)\right\rangle_{\lambda}=\left\langle g(k), k^{\prime}\right\rangle_{\nu}$. The lift of $\left\langle g(k), k^{\prime}\right\rangle_{\nu}$ under $\nu$ is $g(k)$. Under $g, k \mapsto g(k)$. Recall that $g=m^{*}$. Let $c=\in C_{0}^{*}$. Then since $g: K_{0} \rightarrow C_{0}, g(k)=c=m^{*}(c)$. It follows that $m\left(\langle k, c\rangle_{\lambda}\right)=$

\footnotetext{
${ }^{3}$ By use of distinguished triangles, $H_{\mathrm{c}}^{n}\left(c^{o} L\right) \simeq H^{n-1}(L)$ where $\operatorname{dim}(L)=2 n-1$. This implies that $H^{n-1}(L) \rightarrow H^{n}(L)$ are dual since $L$ is compact. Thus, applying Proposition $1.1, H_{\mathrm{c}}^{n}\left(c^{o} L\right) \rightarrow H_{\mathrm{c}}^{n+1}\left(c^{o} L\right)$ is a duality isomorphism. In addition, $\operatorname{dim}\left(Y^{0}\right)=2 n$ so it follows in a straightforward manner that $H^{n}\left(Y^{0}\right)$ and $H_{\mathrm{c}}^{n}\left(Y^{0}\right)$ are dual also.
} 
$\left\langle k, m^{*}(c)\right\rangle_{\lambda}=\langle k, g(k)\rangle_{\lambda}$ where $\langle k, g(k)\rangle_{\lambda} \in C_{0}^{*}$. The lift of $\langle k, g(k)\rangle_{\lambda}=k$. This shows that $m(\lambda(k))\left(k^{\prime}\right)=\nu(g(k))\left(k^{\prime}\right)$.

The final part of showing the diagram commutes is to verify that for $y \in H^{n}\left(i^{*} j_{*} \mathcal{L}_{0}^{*}\right)$ and $c \in C_{0},\langle c, f(y)\rangle_{\nu}=\langle h(c), y\rangle_{\xi}$. This is equivalent to showing that $n(\xi(c))(y)=\xi(h(c))(y)$. Recall that $\xi$ is a map of isomorphism classes. The lift of $\langle h(c), y\rangle_{\xi}=h(c)$. Under $h, c \mapsto h(c)$. Recall that $n=$ $f^{*}$. Let $k \in K^{*}$, then $f(y)=k^{*}(k)$. Consider $\langle c, k\rangle_{\nu} \in K_{0}^{*}$. It follows that $\langle c, f(y)\rangle_{\nu}=\left\langle c, n^{*}(k)\right\rangle_{\nu}\left(\langle c, k\rangle_{\nu}\right)$. Now a lift of $\langle c, k\rangle_{\nu}=c$. This shows that $\langle c, f(y)\rangle_{\nu}=\langle h(c), y\rangle_{\xi}$, and hence the diagram (4.11) commutes.

Corollary 4.1. The perverse sheaf $\underline{\underline{\mathcal{S}_{0}}}$ is self-dual in $\mathbb{P}_{\mathbb{Q}}(Y)$.

Proof. By Theorem 2.1, $\mu: \operatorname{Hom}_{\mathbb{P}}\left(\underline{\underline{\mathcal{S}_{0}}}, \mathcal{D}_{V}\left(\underline{\underline{\mathcal{S}_{0}}}\right)\right) \rightarrow \operatorname{Hom}_{Z}\left(\mu\left(\underline{\underline{\mathcal{S}_{0}}}\right), \mu\left(\mathcal{D}_{V}\left(\underline{\underline{\mathcal{S}_{0}}}\right)\right)\right.$ is a surjection. Since the diagram in $(4.6)$ commutes, $\operatorname{Hom} Z\left(\mu\left(\underline{\underline{\mathcal{S}_{0}}}\right), \overline{\overline{\mu(\mathcal{D}}}{ }_{V}\right.$ $\left.\left.\left(\underline{\underline{\mathcal{S}_{0}}}\right)\right)\right)=\operatorname{Hom}_{Z}\left(\Theta_{0}, \mathcal{D}_{Z}\left(\Theta_{0}\right)\right)$. Now by Theorem 4.1, we have constructed an isomorphism $\Phi \in \operatorname{Hom}_{Z}\left(\Theta_{0}, \mathcal{D}_{Z}\left(\Theta_{0}\right)\right)$. Since $\mu$ is a surjection on morphisms, there exists an isomorphism $\bar{\Phi} \in \operatorname{Hom}_{\mathbb{P}}\left(\underline{\underline{\mathcal{S}_{0}}}, \mathcal{D}_{V}\left(\underline{\underline{\mathcal{S}_{0}}}\right)\right)$. Therefore $\underline{\underline{\mathcal{S}_{0}}}$ is selfdual in $\mathbb{P}_{\mathbb{Q}}(Y)$.

Remark: It is not known whether the isomorphism $\underline{\underline{\mathcal{S}_{0}}} \rightarrow \mathcal{D}_{V}\left(\underline{\underline{\mathcal{S}_{0}}}\right)$ is unique. It is conceivable that there may be several, essentially different, $\overline{\overline{\text { pairings }}} \underline{\underline{\mathcal{S}_{0}}} \otimes$ $\underline{\underline{\mathcal{S}_{0}}} \rightarrow \mathbb{D}_{Y}$. Corollary 4.1 completes the proof of the main result presented $\overline{\overline{\text { in }}}$ Theorem 3.1. A direct corollary to Corollary 4.1 is Poincaré duality. We state this below.

Corollary 4.2 (Poincaré duality). For all degrees $i \geq 0, H^{i}\left(Y ; \underline{\underline{\mathcal{S}_{0}}}\right) \cong H^{2 n-i}$ $\left(Y ; \underline{\underline{\mathcal{S}_{0}}}\right)$.

\section{An example}

\subsection{The construction}

We will look at a very simple construction of space time $\mathcal{M}=X^{3,1} \times Y$ where $Y$ is a single node Calabi-Yau manifold (simple stratified space) as presented on pp. 276-277 in [7]. We will restate this construction here. Consider the family of quintic hypersurfaces in $\mathbb{P}^{4}$, defined by

$$
I:=x_{5}^{3}\left(\Sigma_{i=1}^{4} x_{i}^{2}\right)+\Sigma_{i=1}^{5} a_{i} x_{i}^{5}=0,
$$


where $x_{i}$ are the homogeneous coordinates of $\mathbb{P}^{4}$. On the $x_{5}=0$ hyperplane $\mathbb{P}^{3} \subset \mathbb{P}^{4}$ we have that

$$
\begin{gathered}
I=\Sigma_{i=1}^{4} a_{i} x_{i}^{5}, \\
d I=5 \Sigma_{i=1}^{4} d x_{i} \cdot a_{i} x_{i}^{4} .
\end{gathered}
$$

For generic $a_{i}, I=0=d I$ only at $x_{i}=0$ which is not in $\mathbb{P}^{4}$, so the singular points of $Y$ are all in the $x_{5} \neq 0$ coordinate patch and we set $x_{5}=1$. For generic choice of $a_{i}, I=0$ and $d I=0$ have no common solution, so that the quintic hypersurface $I=0$ in $\mathbb{P}^{4}$ is smooth.

Let $a_{5} \rightarrow 0$. Then, $d I=0$ implies $x_{i}\left(5 a_{i} x_{i}^{3}+2\right)=0$ and candidate singular points $\left(p^{\#}\right)$ are parametrized as

$$
x_{i}=-\xi_{i} \sqrt[3]{\frac{2}{5 a_{i}}} \cdot \omega^{k_{i}}
$$

where $\omega=e^{\frac{2 i \pi}{3}}$ for $k_{i}=0,1,2$, and $\xi_{i}=0,1$ for $i=1,2,3,4$. At these points

$$
I\left(p^{\#}\right)=\frac{3}{5} \sqrt[3]{\frac{4}{25}} \Sigma_{i=1}^{4} \frac{\xi_{i} \omega^{2 k_{i}}}{a_{i}^{\frac{2}{3}}},
$$

the vanishing of which brings about several cases. The case of interest for the purposes of this paper is when $I\left(p^{\#}\right)$ vanishes at a single point, $(0,0,0,0,1) \in \mathbb{P}^{4}$, i.e., setting $\xi_{i}=0 i=1,2,3,4$, whereupon both $I=0$ and $d I=0-$ regardless of the choice of $a_{i}$ 's. There, $\operatorname{det} I^{\prime \prime}=16 \neq 0$ and this singular point is a node.

We now fix some generic choice of $a_{1}, a_{2}, a_{3}, a_{4}$ and regard equation (5.1) as a pencil of quintics in $\mathbb{P}^{4}$, parametrized by $a_{5}$. The quintic hypersurfaces in $\mathbb{P}^{4}$ defined by equation (5.1) for each $a_{5} \neq 0$ and $\left|a_{5}\right|$ not too large ${ }^{4}$ are all smooth; the one at $a_{5}$ is however singular and has a single node. Let $Y_{a_{5}}$ denote these smooth quintics, for $a_{5} \neq 0$; let $Y=Y_{0}$ denote the 1-node singular quintic, at $a_{5}=0$.

\subsection{Computing $H^{*}(Y)$}

We now focus on the quintic with a single node, $Y=Y_{0}$ where $Y^{o}$ denotes the non-singular part of $Y$. Let $\widetilde{Y}$ be the small resolution of $Y$; there is no obstruction to local surgery, replacing the node by a $\mathbb{P}^{1}$. Note that $\widetilde{Y}$

\footnotetext{
${ }^{4}$ Given concrete values of $a_{1}, a_{2}, a_{3}, a_{4}$, there may well exist an upper bound on $\left|a_{5}\right|$ for this to be true.
} 
cannot be Kähler: any putative Kähler form would have to be null on the exceptional $\mathbb{P}^{1}[7] .^{5}$

We want to compute $H^{*}$ using $\mathbb{S}_{0}$ and compare it to the results obtained from string theory. For $n \neq 3, H^{n}\left(Y ; \underline{\underline{\mathbb{S}_{0}}}\right) \simeq H^{n}(Y ; \underline{\underline{I C}}) \simeq H^{n}(\widetilde{Y} ; \underline{\underline{\mathbb{Q}}})$ where $\widetilde{Y}$ is the small resolution. In the middle dimension we must use part 2 (short exact sequences) of Theorem 3.1. Recall that $H^{n}\left(Y ; \underline{\underline{\mathcal{S}_{0}}}\right)$ is specified by the following two canonical short exact sequences:

1. $0 \rightarrow K_{0} \rightarrow H^{n}\left(Y ; \underline{\mathcal{S}_{0}}\right) \rightarrow H^{n}\left(Y^{o}\right) \rightarrow 0$,

2. $0 \rightarrow H_{\mathrm{c}}^{n}\left(Y^{o}\right) \rightarrow H^{\overline{\bar{n}}}\left(Y ; \underline{\underline{\mathcal{S}_{0}}}\right) \rightarrow C_{0} \rightarrow 0$,

where $K_{0}=\operatorname{Im}\left(H_{\mathrm{c}}^{n}\left(c^{o} L\right) \rightarrow H_{\mathrm{c}}^{n}\left(Y^{o}\right)\right), C_{0}=\operatorname{Im}\left(H^{n}\left(Y^{o}\right) \rightarrow H_{\mathrm{c}}^{n+1}\left(c^{o} L\right)\right)$ with the non-singular part $Y^{o}$ and $L=S^{2} \times S^{3}$ the link of the singular point. In addition, $H^{n}(Y ; \underline{\underline{Q}}) \simeq H_{\mathrm{c}}^{n}\left(Y^{o} ; \underline{\underline{Q}}\right), H_{\mathrm{c}}^{n}\left(c^{o} L ; \underline{\underline{Q}}\right) \simeq H^{n}(L ; \underline{\underline{Q}})$ and $H^{n}(\widetilde{\widetilde{Y}} ; \underline{\underline{\mathbb{Q}}}) \simeq$ $H^{n}(Y ; \underline{\underline{I C}})$.

\subsection{String theoretic description}

The analysis of Type IIB superstring compactification on $Y$ à la Strominger [5] would proceed as a limiting process, starting from some $Y_{a_{5} \neq 0}$, and taking the limit $a_{5} \rightarrow 0$. For $\left|a_{5}\right|$ sufficiently small, $Y_{a_{5} \neq 0}$ is a smooth quintic and a Type IIB superstring compactification would feature one massless vector supermultiplet of $N=2$ supersymmetry in the effective four-dimensional spacetime, and 101 massless hypermultiplets plus their Hermitian conjugates. In the limit $a_{5} \rightarrow 0$ one of these hypermultiplets and its Hermitian conjugate would be "frozen" to a constant value and become removed from the spectrum of massless (variable) fields. However, Strominger showed that a massless state and its Hermitian conjugate would become massless in the $a_{5} \rightarrow 0$ limit where $\left|a_{5}\right|$ is small enough, $Y_{a_{5} \neq 0}$ contains a "vanishing" $S^{3}$, which shrinks to a point in the $a_{5} \rightarrow 0$ limit. Strominger's replacement state turns out to have a mass proportional to the volume of the vanishing $S^{3}$. This is massive in compactifications on $Y_{a_{5} \neq 0}$, but becomes massless in compactifications on $Y=Y_{0}$.

\footnotetext{
${ }^{5}$ The single node example presented here has a major drawback for application in superstring theory: neither $Y=Y_{0}$ nor its small resolution $\widetilde{Y}$ admit a Kähler metric. However, the purpose of this example is to demonstrate that the complex of sheaves $\underline{\underline{\mathbb{S}_{0}}}$ can provide the needed ranks in all dimensions (as suggested by Hubsch [8] and Strominger $[5])$.
} 
Table 1: Dimension of $H^{n}(Y ; *)$

\begin{tabular}{lccc}
\hline $\operatorname{Deg}$ & $H^{n}\left(Y ; \underline{\underline{\mathbb{S}_{0}}}\right)$ & $H^{n}(Y ; \underline{\underline{\mathbb{Q}}})$ & $H^{n}(\tilde{Y} ; \underline{\underline{\mathbb{Q}}})$ \\
\hline 6 & 1 & 1 & 1 \\
5 & 0 & 0 & 0 \\
4 & 1 & 2 & 2 \\
3 & 204 & 203 & 202 \\
2 & 1 & 1 & 2 \\
1 & 0 & 0 & 0 \\
0 & 1 & 1 & 1 \\
\hline
\end{tabular}

Therefore, the spectrum of massless fields in Type IIB superstring compactifications on $Y_{a_{5}}$ remains constant in the $a_{5} \rightarrow 0$ limit which can be obtained from Table 1:

$$
\operatorname{dim} H^{n}\left(Y ; \underline{\underline{\mathbb{S}_{0}}}\right)=\operatorname{dim} H^{n}\left(Y_{a_{5} \neq 0} ; \underline{\underline{\mathbb{Q}}}\right) .
$$

Note that in singular 3-folds with more than one node that do have a Kähler small resolution, the $\mathbb{S}_{0}$-valued cohomology of the singular model would

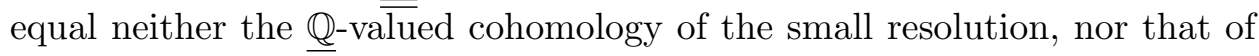
the smooth deformation. The equality (5.6) owes to the fact that $Y$ has a single node, whereupon its small resolution cannot be Kähler.

\section{Final remarks}

In this paper we presented the construction and properties of a self-dual perverse sheaf $\underline{\underline{\mathcal{S}_{0}}}$ using techniques of MacPherson-Vilonen [2]. In addition, this perverse sheaf was shown to satisfy Poincarè duality (property (3) of the Kähler package) as well as having relevant applications for cases of Type IIB superstring compactification. It is currently not known whether the remainder of the Kähler package holds for $\underline{\underline{\mathcal{S}_{0}}}$. These are currently open problems.

\subsection{The Kähler package}

Proofs of all four parts of the Kähler package were not addressed in this paper. Only Corollary 4.2, which was a consequence of the self-duality of $\underline{\underline{\mathcal{S}_{0}}}$, provided Poincare duality. It would take additional effort to prove the remaining parts of the Kähler package. The difficulties would lie in proving 
Hodge decomposition and the Kunneth formula. It is not clear if there is a pure or mixed Hodge structure of $\underline{\underline{\mathcal{S}_{0}}}$. This has to be explored.

\subsection{Multiple singular points}

There are cases that arise in String theory where the target space will have more than one singularity. We would like to extend this effort (one singular point) to include the case of multiple singular points. Although this may seem to be a straightforward transition, there appear to be problems in preserving some of the properties of the needed maps.

In the case of multiple singular points, the singularity $\{y\}$ now becomes a singular set made up of isolated distinct singular points $\Sigma=\left\{y_{1}, \ldots, y_{r}\right\}$ where $r$ is the number of singular points. As a result, the injection $j$ now becomes $\hat{j}: \Sigma \hookrightarrow Y$ while $i: Y^{o} \rightarrow Y$ remains unchanged. Consequently, the maps in the long exact sequences that arise from the distinguished triangles are altered by the inclusion of more singular points. In order to qualify this difficulty we can begin by restating the last three identities of Lemma 1.2 as follows.

Lemma 6.1. Let $Y$ be a simple stratified space and let $\underline{\underline{\mathcal{F}}}$ be a complex of sheaves on $Y$. Let $\Sigma=\left\{y_{1}, \ldots, y_{r}\right\} \in Y$ be the singular set and let $U_{y_{b}}$ be a distinguished neighborhood of $y_{b}$ so that $\bar{U}_{y_{b}} \simeq c L_{y_{b}}$ and $U_{y_{b}} \simeq c^{o} L_{y_{b}}$ for $1 \leq b \leq r$. Let $i: Y^{o} \hookrightarrow Y$ and $\hat{j}: \Sigma \hookrightarrow Y$ be inclusions. Then there are natural isomorphisms

1. $H^{m}\left(Y ; \hat{j}_{*} \hat{j}^{*} \underline{\underline{\underline{\mathcal{F}}}}\right) \cong \bigoplus_{b=1}^{r} H^{m}\left(U_{y_{b}} ; \underline{\underline{\mathcal{F}}}\right), \forall m>0$,

2. $H^{m}\left(Y ; \hat{j}_{*} \hat{j}^{\prime} \underline{\underline{\underline{\mathcal{F}}}}\right) \cong \bigoplus_{b=1}^{r} H_{\mathrm{c}}^{m}\left(U_{y_{b}} ; \overline{\underline{\underline{\mathcal{F}}}}\right), \forall m>0$,

3. $H^{m}\left(Y ; \hat{j}^{*} i_{*} \underline{\underline{\mathcal{F}}}\right) \cong \bigoplus_{b=1}^{r} H^{m}\left(L_{y_{b}} \times(0,1) ; \underline{\underline{\mathcal{F}}}\right), \forall m>0$ where $L_{y_{b}}$ is the link of the singular point $y_{b}$ for $1 \leq b \leq r$.

(Remark: Note that $U_{y_{b}} \cong c L_{y_{b}}$ where $c L_{y_{b}}$ is the cone over the link of the singular point $y_{b}$.)

Consider the distinguished triangles in diagrams (1.3) and (1.4) applied to $\underline{\underline{\mathcal{S}_{0}}}$. Rewriting these triangles using $\hat{j}: \Sigma \hookrightarrow Y$ instead of $j:\{y\} \hookrightarrow Y$ we obtain

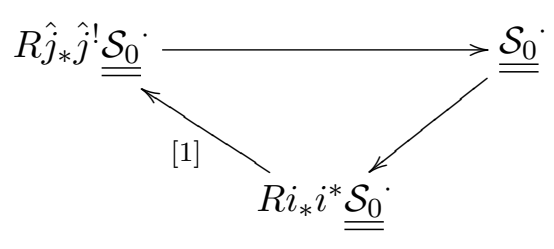




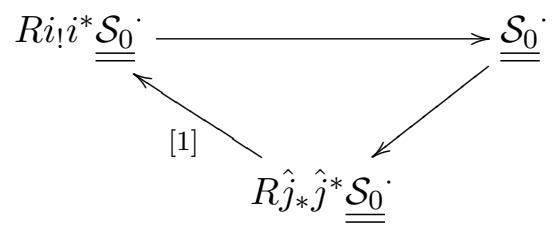

The resulting long exact sequence in degree $n$ for the triangle in diagram (6.1) gives

$$
\begin{aligned}
\cdots \longrightarrow & \bigoplus_{b=1}^{r} H_{\mathrm{c}}^{n}\left(c L_{y_{b}} ; \underline{\underline{\mathcal{S}_{0}}}\right) \longrightarrow H^{n}\left(Y ; \underline{\underline{\mathcal{S}_{0}}}\right) \longrightarrow H^{n}\left(Y^{o}\right) \\
\longrightarrow & \bigoplus_{b=1}^{r} H_{\mathrm{c}}^{n+1}\left(c L_{y_{b}} ; \underline{\underline{\mathcal{S}_{0}}}\right) \longrightarrow \cdots
\end{aligned}
$$

Let the maps of the long exact sequence in equation (6.3) be defined as

$$
\begin{aligned}
& \alpha_{1}: \bigoplus_{b=1}^{r} H_{\mathrm{c}}^{n}\left(c L_{y_{b}} ; \underline{\underline{\mathcal{S}_{0}}}\right) \rightarrow H^{n}\left(Y ; \underline{\underline{\mathcal{S}_{0}}}\right) \\
& \beta_{1}: H^{n}\left(Y ; \underline{\underline{\mathcal{S}_{0}}}\right) \rightarrow H^{n}\left(Y^{o}\right) \\
& \gamma_{1}: H^{n}\left(Y^{o}\right) \rightarrow \bigoplus_{b=1}^{r} H_{\mathrm{c}}^{n+1}\left(c L_{y_{b}} ; \underline{\underline{\mathcal{S}_{0}}}\right)
\end{aligned}
$$

Similarly the resulting long exact sequence in degree $n$ for the triangle in diagram (6.2) gives

$$
\begin{aligned}
\cdots \longrightarrow & \bigoplus_{b=1}^{r} H^{n-1}\left(c L_{y_{b}} ; \underline{\underline{\mathcal{S}_{0}}}\right) \longrightarrow H_{\mathrm{c}}^{n}\left(Y^{o}\right) \longrightarrow H^{n}\left(Y ; \underline{\underline{\mathcal{S}_{0}}}\right) \\
\longrightarrow & \bigoplus_{b=1}^{r} H^{n}\left(c L_{y_{b}} ; \underline{\underline{\mathcal{S}_{0}}}\right) \longrightarrow \cdots
\end{aligned}
$$

Similarly let the maps of the long exact sequence in equation (6.7) be defined as

$$
\begin{aligned}
& \alpha_{2}: \bigoplus_{b=1}^{r} H^{n-1}\left(c L_{y_{b}} ; \underline{\underline{\mathcal{S}_{0}}}\right) \rightarrow H_{\mathrm{c}}^{n}\left(Y^{o}\right) \\
& \beta_{2}: H_{\mathrm{c}}^{n}\left(Y^{o}\right) \rightarrow H^{n}\left(Y ; \underline{\underline{\mathcal{S}_{0}}}\right) \\
& \gamma_{2}: H^{n}\left(Y ; \underline{\underline{\mathcal{S}_{0}}}\right) \rightarrow \bigoplus_{b=1}^{r} H^{n}\left(c L_{y_{b}} ; \underline{\underline{\mathcal{S}_{0}}}\right)
\end{aligned}
$$


Recall the figure where the map $c$ is an injection and $d$ is a surjection that was shown to exist in the proof of Theorem 3.1. These were requirements imposed by String theory (see Section 1.3 for a discussion).

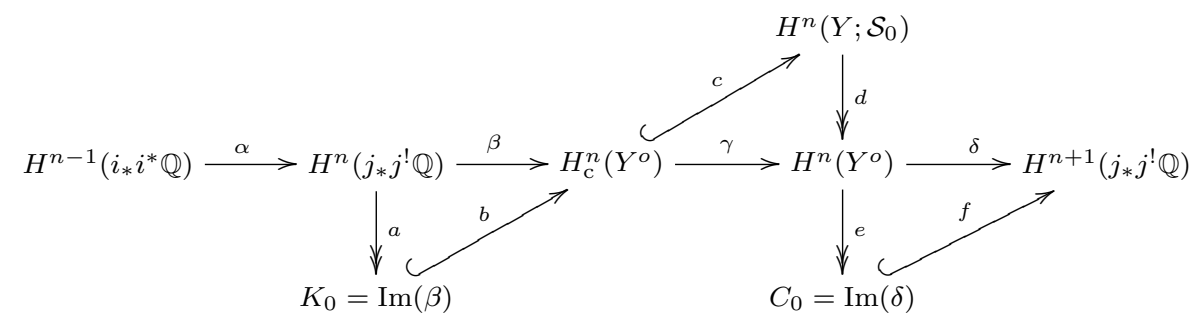

Equation (6.11) and its maps were defined in the case of one singular point where the map $j:\{p t\} \rightarrow Y$. Now if we replace $j$ with $\hat{j}: \Sigma \rightarrow Y$ it is not completely clear if the map $c$ will remain an injection and $d$ will remain a surjection. (Recall that in order to obtain a cohomology for String theory it is a requirement that the map $c$ be injective and $d$ be surjective.) These are necessary conditions in order to obtain a larger rank of cohomology in the middle dimension. Notice that in the long exact sequences using $\hat{j}$, equations (6.3) and (6.7), if we replaces $j$ by $\hat{j}$, the maps $c$ and $d$ can be described as follows:

$c$ corresponds to the map $\beta_{2} \Rightarrow \alpha_{2}$ needs to be the 0-map.

$d$ corresponds to the map $\beta_{1} \Rightarrow \gamma_{1}$ needs to be the 0 -map.

It is not quite clear however, if for all singular points in $\Sigma$ the maps $\alpha_{2}$ and $\gamma_{1}$ can be made to be the 0 -maps. This is currently an obstruction to extending the singular point case to the case of a singular set. Hopefully a future effort will address this.

\section{Acknowledgments}

The author wishes to extend special thanks to R.M Goresky and T. Hubsch for the very helpful discussions and detailed reviews of the final manuscript. The author also wishes to thank D. Massey for helpful discussions concerning perverse sheaves and a review of the initial manuscript. Many thanks to R. MacPherson for making the initial remark that there was such a perverse sheaf that had the properties we were looking for. The Institute for Advanced Study, Princeton, NJ deserves many compliments for its hospitality and use of facilities during the development of this work. 


\section{References}

[1] T. Hubsch, On a stringy singular cohomology, Mod. Phys. Lett. A12 (1997), 521-533, (hep-th/9612075).

[2] R. MacPherson and K. Vilonen, Elementary constructions of perverse sheaves, Inv. Math. 84 (1986), 403-435.

[3] P.S. Green and T.Hubsch, Possible phase transitions among Calabi-Yau compactifications, Phys. Rev. Lett. 61 (1988), 1163-1167.

[4] P.S. Green and T. Hubsch, Connecting moduli spaces of Calabi-Yau threefolds, Commun. Math. Phys. 119 (1988), 431-441.

[5] A. Strominger, Massless black holes and conifolds in string theory, Nucl. Phys. B451 (1995), 96-108, (hep-th/9504090).

[6] T. Hubsch and A. Rahman, On the geometry and homology of certain simple stratified varieties, J. Geom. Phys. 53 (2005), 31-48, (math.AG/0210394).

[7] T. Hubsch, Calabi-Yau manifolds: a bestiary for physicists, World Scientific Pub Co., Singapore, 1992.

[8] T. Hubsch, How singular a space can superstrings thread?, Mod. Phys. Lett. A6 (1991), 207-216.

[9] M. Goresky and R. MacPherson, Intersection homology II, Inv. Math. 71 (1983), 77-129.

[10] J. Cheeger, M. Goreksy and R. MacPherson, L2-cohomology and intersection homology of singular algebraic varieties, Seminar on Differential Geometry, S.T. Yau ed. Ann. Math. Stud. 102 (1982), 303-340.

[11] M. Goresky and R. MacPherson, Intersection homology theory, Topology 19 (1980) 135-162.

[12] M. Kashiwara and P. Schapira, Sheaves on manifolds, Springer-Verlag, Berlin, 1980.

[13] A. Dimca, Sheaves in topology, Springer, Berlin, 2004.

[14] S.I. Gelfand and Y.I. Manin, Homological algebra, Springer, Berlin, 1999.

[15] J.L. Verdier, Dualitè dans la cohomologie des espaces localment compactes, Seminar Bourbaki 300 (1965).

[16] B. Iversen, Cohomology of sheaves, Aarhus universitet, Matematisk Institut, 1984.

[17] R.M. Goresky, G. Harder and R. MacPherson, Weighted cohomology, Inv. Math. 116 (1994), 139-213. 
